\title{
Social Media and Public Outreach: A Physician Primer
}

\author{
A. Radmanesh, R. Duszak, and R.T. Fitzgerald
}

$\mathbf{T}$ he emergence of social media with easy and anywhere smartphone access has facilitated our society's information-craving behaviors to the point that many rely on the Web and social media to make health-related decisions. Recognizing this reality, many health care professionals and organizations have in recent years become increasingly visible on social media.

According to a database of health care organizations actively using social media, over 1500 US hospitals now have a social media presence. In California alone, 105 hospitals sponsor 444 social media accounts that include 53 YouTube accounts, 89 Facebook accounts, 69 Twitter accounts, 39 LinkedIn accounts, 78 Foursquare accounts, and 11 blogs. ${ }^{1}$

Many social media pages and on-line review sites used by patients are unfortunately vulnerable to bias and often inaccurate. Given the role of social media in forming the public's "image" of health care facilities and providers, a credible presence by those parties is more important than ever, particularly as crowdsourced metrics potentially take root as a measure of quality. ${ }^{2}$

Patients should always be the primary focus of our work. To help shape ongoing reform efforts, the American College of Radiology recently launched the Imaging 3.0 initiative. $^{3}$ One of its many goals is to improve patient awareness of the integral role of radiologists in their overall health care, and to enhance their understanding of and comfort with the tests and procedures they are undergoing. Social media can be a major facilitator of that culture shift.

The responsible use of social media provides golden opportunities for marketing physician services, as well as for contributing to public health by providing high quality on-line content that is both accurate and understandable to laypeople. Radiologists, for example, can leverage social media to help correct widespread and

From the Department of Radiology and Biomedical Imaging (A.R.), University of California, San Francisco, San Francisco, California; Department of Radiology (R.D.) Emory University School of Medicine, Atlanta, Georgia; Harvey L. Neiman Health Policy Institute (R.D.), Reston, Virginia; and Department of Radiology (R.T.F.), University of Arkansas, Little Rock, Arkansas.

Please address correspondence to Alireza Radmanesh, MD, Department of Radiology and Biomedical Imaging, University of California, San Francisco, 505 Parnassus Ave, L-352, San Francisco, CA 94143; e-mail: alireza.radmanesh@ucsf.edu; (AliRadmanMD

http://dx.doi.org/10.3174/ajnr.A4100 frequently publicized fear-generating myths regarding the risks of ionizing radiation from diagnostic imaging.

Public perception of who we are and what we do is the engine of professional advocacy campaigns and important to the future of radiology as a specialty. Social media engagement with patients, their employers, policymakers, and lawmakers can be a strong vehicle to help accurately form that perception. A current example pertains to Medicare reimbursement for lung cancer screening. The June 2014 Journal of the American College of Radiology (\#JACR) tweet-chat engaged both physicians and patients, and was focused on lung cancer screening - a hot topic with many public policy considerations - with an emphasis on patient experience. A tweet-chat is a live Twitter event that is usually moderated and focused around a general topic. To filter all the chatter on Twitter into a single conversation, a hashtag (\#) is used. A set time is also established so that the moderator, guest, or host is available to engage in the conversation. ${ }^{4}$

Despite its enormous potentials, the boundless reach of social media creates legal and ethical challenges for physicians. The "4sphere" concept provides a simple guiding framework for professional users of social media to avoid undesired ethical and legal consequences. ${ }^{5}$ It dictates that any social media activity should be in compliance with the media site's terms of service, professional standards, organizational policies, and individual best practices (Fig 1). To those ends, resources are available. Physicians are advised to familiarize themselves with policies developed by their health care organizations to address concerns of reputation, privacy, and productivity. The American Medical Association has published its own "Professionalism in the Use of Social Media" guidelines document. ${ }^{6,7}$

Social media and on-line platforms are here to stay. Some platforms may fade away, but new ones will emerge. Based on current trends, media skills could eventually become a professional technical competency and a component of future medical education curricula.

Social media are new to many currently practicing physicians and that may generate anxiety. The avoidance of social media, however, does not protect us as professionals from our public. On the contrary, it constrains our on-line identity and cedes its control to nameless and often uninformed sources. We believe it is far better to be in control- to the degree we can-of what our pa- 


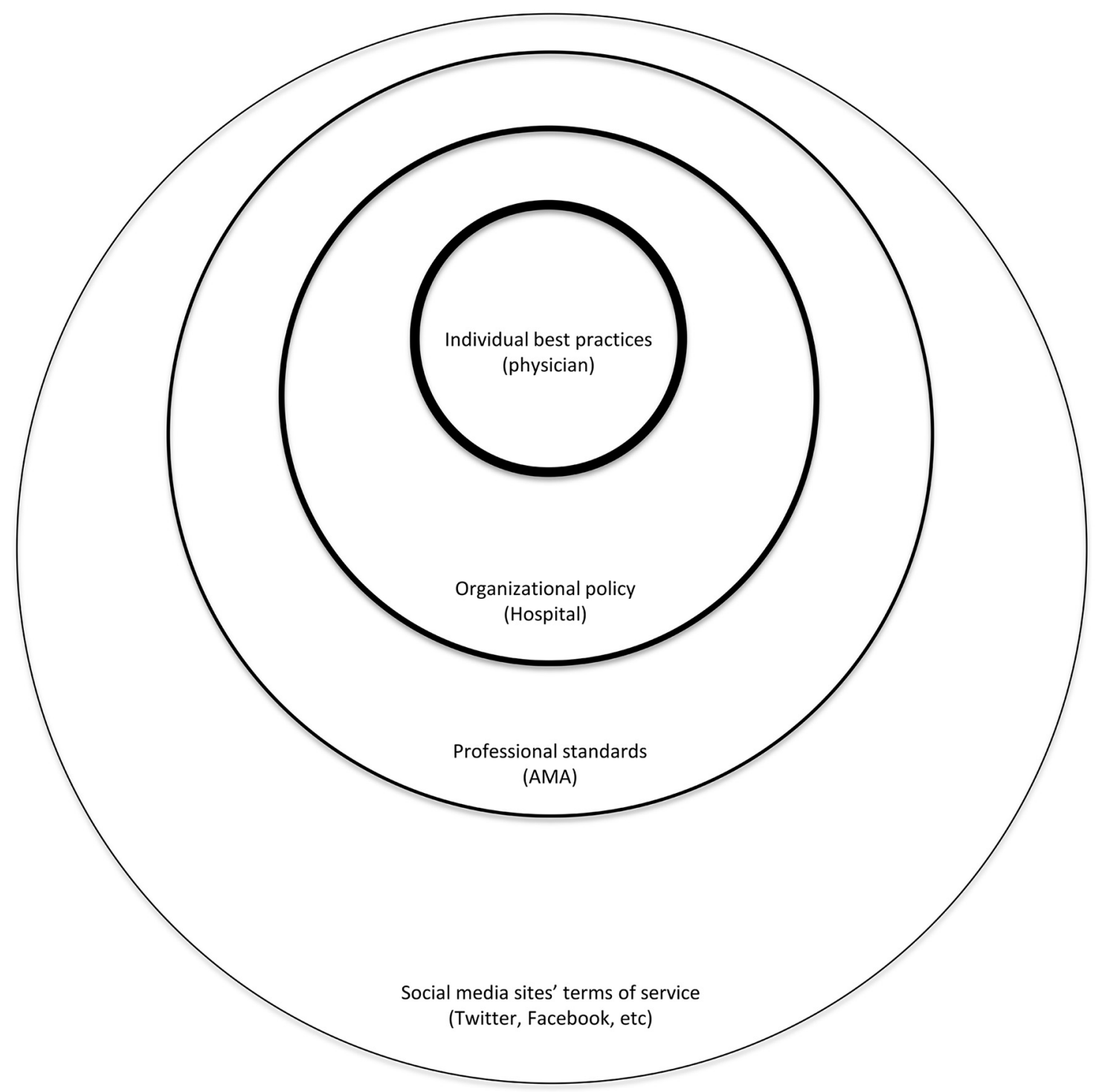

FIG 1. "Four-sphere" concept as a guiding framework for professional use of social media. Adapted from Gagnon et al. ${ }^{5}$

tients, colleagues, and others find about us using search engines and other on-line tools. At the end of the day, as our on-line and real identities increasingly converge, they together define who we are in the eyes of others.

\section{REFERENCES}

1. Mayo Clinic Center for Social Media. Health care social media list. http:// network.socialmedia.mayoclinic.org/hcsml-grid/. Accessed July 5, 2014

2. Timian A, Rupcic S, Kachnowski S, et al. Do patients "like" good care? Measuring hospital quality via Facebook. Am J Med Qual 2013;28:374-82

3. Ellenbogen PH. Imaging 3.0: what is it? J Am Coll Radiol 2013;10:229
4. Cooper S. The ultimate guide to hosting a tweet chat. Forbes. September 30, 2013. http://www.forbes.com/sites/stevecooper/2013/09/ 30/the-ultimate-guide-to-hosting-a-tweet-chat/. Accessed July 2, 2014

5. Gagnon K, Sabus C. Professionalism in a digital age: opportunities and considerations for using social media in health care. Phys Ther 2015;95:406-14

6. American Medical Association. Opinion 9.124_professionalism in the use of social media. June 2011. http://www.ama-assn.org/ama/ pub/physician-resources/medical-ethics/code-medical-ethics/opinion9124. page? Accessed July 8, 2014

7. Shore R, Halsey J, Shah K, et al. Report of the AMA council on ethical and judicial affairs: professionalism in the use of social media. JClin Ethics 2011;22:165-72 